ARTICLE

DOI: $10.1038 / s 41467-018-03968-5 \quad$ OPEN

\title{
Culturing of female bladder bacteria reveals an interconnected urogenital microbiota
}

\author{
Krystal Thomas-White ${ }^{1}$, Samuel C. Forster (10 2,3,4, Nitin Kumar ${ }^{2}$, Michelle Van Kuiken ${ }^{5}$, Catherine Putonti $6,7,8$, \\ Mark D. Stares ${ }^{2}$, Evann E. Hilt', Travis K. Price ${ }^{1}$, Alan J. Wolfe ${ }^{1} \&$ Trevor D. Lawley ${ }^{2}$
}

Metagenomic analyses have indicated that the female bladder harbors an indigenous microbiota. However, there are few cultured reference strains with sequenced genomes available for functional and experimental analyses. Here we isolate and genome-sequence 149 bacterial strains from catheterized urine of 77 women. This culture collection spans 78 species, representing approximately two thirds of the bacterial diversity within the sampled bladders, including Proteobacteria, Actinobacteria, and Firmicutes. Detailed genomic and functional comparison of the bladder microbiota to the gastrointestinal and vaginal microbiotas demonstrates similar vaginal and bladder microbiota, with functional capacities that are distinct from those observed in the gastrointestinal microbiota. Whole-genome phylogenetic analysis of bacterial strains isolated from the vagina and bladder in the same women identifies highly similar Escherichia coli, Streptococcus anginosus, Lactobacillus iners, and Lactobacillus crispatus, suggesting an interlinked female urogenital microbiota that is not only limited to pathogens but is also characteristic of health-associated commensals.

\footnotetext{
${ }^{1}$ Department of Microbiology and Immunology, Stritch School of Medicine, Loyola University Chicago, Maywood, IL 60153, USA. ${ }^{2}$ Host-Microbiota Interactions Laboratory, Wellcome Sanger Institute, Hinxton CB10 1SA, UK. ${ }^{3}$ Centre for Innate Immunity and Infectious Diseases, Hudson Institute of Medical Research, Clayton, VIC 3168, Australia. ${ }^{4}$ Department of Molecular and Translational Sciences, Monash University, Clayton VIC 3800, Australia. ${ }^{5}$ Department of Urology, Stritch School of Medicine, Loyola University Chicago, Maywood, IL 60153, USA. ${ }^{6}$ Department of Biology, Loyola University Chicago, Chicago, IL 60660, USA. ${ }^{7}$ Department of Computer Science Loyola University Chicago Chicago IL 60660 USA. ${ }^{8}$ Bioinformatics Program Loyola University Chicago Chicago IL 60660 USA. These authors contributed equally: Krystal Thomas-White, Samuel C. Forster, Nitin Kumar. These authors jointly supervised this work: Alan J. Wolfe, Trevor D. Lawley. Correspondence and requests for materials should be addressed to A.J.W. (email: awolfe@luc.edu) or to T.D.L. (email: t12@sanger.ac.uk)
} 
C ontrary to medical dogma, urine is not sterile, even in asymptomatic individuals ${ }^{1-5}$. For over 60 years, the standard urine culture protocol has represented the primary tool for detecting bacteria in clinical microbiology laboratories. This aerobic protocol was designed to detect causative agents of pyelonephritis ${ }^{6}$ and is particularly effective at detecting abundant Escherichia coli $\left(>10^{5}\right.$ colony-forming unit $\left.(\mathrm{CFU}) / \mathrm{ml}\right)$ but little else $^{7}$. Culture-independent analysis of urine obtained by suprapubic aspirate, which bypasses the vulva, vagina, and urethra, demonstrates the presence of bacteria in the bladders of women ${ }^{1}$. The microbial profiles observed in aspirated urine are similar to those in urine obtained by transurethral catheter (TUC), indicating the avoidance of urethral contamination in catheterized samples ${ }^{1}$. We note the difference between TUC, which is transient and not associated with catheter-associated urinary tract infection (CAUTI), and indwelling catheters that are known to harbor biofilms and result in CAUTI. Analyzing urine sampled by TUC, we previously found that the majority of asymptomatic women contain bacterial genera typically not detected by standard urine culture ${ }^{1,3}$. These genera, including Lactobacillus, Gardnerella, Streptococcus, Staphylococcus, and Corynebacterium, tend to be in low abundance (between 10 and $\left.10^{4} \mathrm{CFU} / \mathrm{ml}\right)^{3}$ and require growth conditions and carbon sources not available in standard urine culture ${ }^{2,8}$. Therefore, we developed an expanded quantitative urine culture (EQUC) protocol that captures a broad range of bacterial taxa ${ }^{3}$.

Previous studies, using EQUC in combination with 16S rRNA sequencing ${ }^{2,3,9}$, suggest the bladder microbiota of asymptomatic women typically contains low bacterial diversity, with increases in diversity indicative of urgency urinary incontinence symptoms and a decreased response to anticholinergic medication ${ }^{2,9,10}$. They also highlight bacterial species (e.g., Streptococcus anginosus and Gardnerella vaginalis) that are associated with urgency urinary incontinence symptoms and others (e.g., Lactobacillus crispatus) that are associated with the lack of lower urinary tract symptoms ${ }^{2}$. Evidence also exists that women with communities dominated by specific Lactobacillus species are less likely to develop post-instrumentation and postoperative urinary tract infections (UTIs) ${ }^{8,11}$. However, owing to the lack of bladderspecific bacterial reference genomes, high-resolution taxonomic characterization to the bacterial strain level, functional analysis, and comparison to other microbiota communities remain to be performed.

In the present study, we combined EQUC with large-scale whole-genome sequencing to comprehensively characterize the microbiota composition of the female bladder in symptomatic and asymptomatic peri-menopausal women. We compared this new culture collection to previously collected gut and vaginal collections and identified similarities between the bladder and vagina but not the gut. Finally, we identified highly similar species that reside in both the bladder and vagina of individual women indicative of interconnected urogenital microbiota.

\section{Results}

The bladder microbiota culture collection. Overall, we archived and genome-sequenced 149 isolates representing 3 phyla, 7 classes, 11 orders, 23 families, 36 genera, and 78 species (Fig. 1; Supplementary Data 1). These organisms were isolated from 38 asymptomatic individuals (67 isolates) and 39 symptomatic individuals (82 isolates) (Supplementary Data 1). Uropathogenic species, such as E. coli, Klebsiella pneumoniae, Proteus mirabilis, Enterobacter cloaceae, Morganella morganii, and Pseudomonas aeruginosa, represented only $7.7 \%$ (6/78) of the phylogenetic diversity cultivatable from the bladder. In fact, other than these uropathogens, very few Proteobacteria or even Gram-negative organisms were found. Instead, the largest number of isolated species was from the Gram-positive phyla Firmicutes $(47.4 \%$, 37/ $78)$ and Actinobacteria (38.5\%, 30/78), particularly the families Streptococcaceae $(11.0 \%, 9 / 78)$, Lactobacillaceae $(11.0 \%, 9 / 78)$, Corynebacteriaceae $(10.3 \%, 8 / 78)$, and Actinomycetaceae $(10.3 \%$, $8 / 78)$. To understand the extent of the total bacterial community represented by this culture collection, we next undertook wholegenome metagenomic sequencing on 12 samples. This analysis suggests that EQUC captures approximately $66.4 \%$ of bacterial abundance within the bladder microbiota representing approximately $72.0 \%$ of the genera (Supplementary Table 1 ). The only genera detected by metagenomics without representative strains within our culture collection were anaerobes from the phyla Actinobacteria (Propionimicrobium, Varibaculum, and Atopobium), Firmicutes (Peptoniphilus, Megasphaera, Finegoldia), and Bacteroidetes (Prevotella).

Comparison of bladder, gut, and vaginal isolates. To place the bladder microbiota in the context of other well-studied body sites, we compared our bladder genome collection with strains from 67 publicly available vaginal (Supplementary Table 2) and 120 gastrointestinal (Supplementary Table 3) species cultivated from unrelated healthy women. Based on whole-genome pairwise average nucleotide identity $(\mathrm{ANI})^{12-14}$, only one species, Bifidobacterium bifidum, was detected in all three body sites (pairwise ANI $>95 \%$, indicating same species). In contrast, 23 species were found in both the bladder and vaginal microbiota (ANI > 95\%). We also identified seven species typically associated with UTIs ( $E$. cloacae, E. coli, P. aeruginosa, Bacillus infantis, K. pneumoniae, Gardnerella terrae, and Bacillus idriensis), with three species isolated only from the bladder of symptomatic women (P. aeruginosa, K. pneumoniae, and E. cloacae) (Supplementary Table 4). Remarkably, from unrelated women, four species were identified (Actinomyces neuii, Lactobacillus crispatus, L. gasseri, and L. jensenii) that were highly similar between the bladder and vagina (Supplementary Table 5).

Protein-coding functions of bladder isolates from asymptomatic individuals. To determine the protein functions encoded by the genomes of members of the healthy bladder microbiota and its relationship to the functions encoded by the genomes of microbiota from other body sites, we analyzed the genomes of bladder strains isolated from asymptomatic women, with gastrointestinal and vaginal strains isolated from other asymptomatic individuals. Applying conserved domain database (CDD) ${ }^{15}$ and discriminant analysis of principle components (DAPC) ${ }^{16}$, we compared the protein domains of the 67 bladder strains from healthy women with protein domains of existing 92 publicly available vaginal and 152 gastrointestinal strains cultivated from unrelated healthy individuals ${ }^{17,18}$. Consistent with the species analysis, this comparison demonstrates clear overlapping protein functions within the bladder and vaginal strains that were largely separated from protein functions found in the gastrointestinal strains (Fig. 2). Taken together, these results indicate the presence of shared functions across the bladder and vaginal microbiota that are clearly distinct from those of gastrointestinal species.

To understand the characteristics that differentiate vaginal and bladder bacteria from gastrointestinal tract bacteria, we next performed functional genomic comparisons. Applying Clusters of Orthologous Groups of proteins (COG) analysis, we identified significant enrichment of functional domains in the urogenital-associated bacteria associated with almost the entire mevalonate-dependent pathway for isoprenoid biosynthesis (Supplementary Table 6): 3-hydroxy-3-methylglutaryl CoA synthase $\left(q<1.33 \times 10^{-18}\right), \quad 3$-hydroxy-3-methylglutaryl CoA 


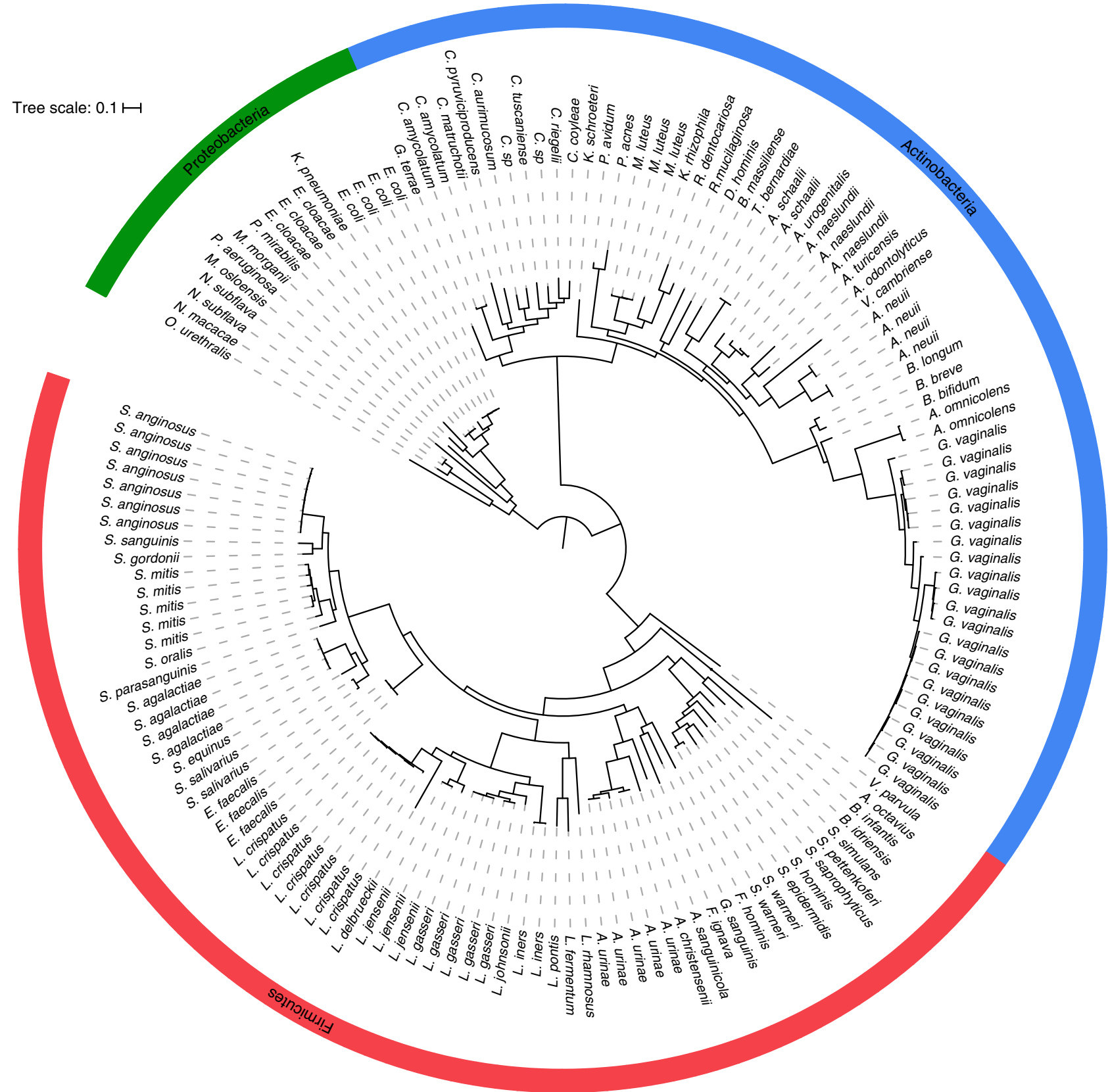

Fig. 1 Phylogenetic tree representing diversity of bacteria cultured from the female bladder. A representation of the full bacterial diversity ( $n=149$ isolates) that can be isolated using the expanded quantitative urine culture (EQUC) method from catheterized urine samples $(n=77$ patients)

reductase $\left(q<5.17 \times 10^{-11}\right)$, mevalonate kinase $\left(q<5.84 \times 10^{-14}\right)$, and mevalonate pyrophosphate decarboxylase $\left(q<8.15 \times 10^{-15}\right)$. Also enriched was pantothenate kinase, a protein involved the biosynthesis of the coenzyme A required for isoprenoid biosnthesis $\left(q<1.1 \times 10^{-10}\right)$. Other enriched functional domains included several transport systems [the permease component of a predicted ABC-type exoprotein transport system $\left(q<5.71 \times 10^{-15}\right)$ and L-asparagine transporter and permeases $\left.\left(q<1.40 \times 10^{-13}\right)\right]$ and a component of the $\mathrm{Co} / \mathrm{Zn} / \mathrm{Cd}$ efflux system $\left(q<2.53 \times 10^{-13}\right)$ involved in metal resistance. Also enriched were protoheme ferro-lyase $\left(q<4.40 \times 10^{-14}\right)$, the luciferase family of flavin-dependent oxidoreductases $(q<2.74 \times$ $\left.10^{-13}\right)$, NAD(P)H-dependent FMN reductase $\left(q<6.52 \times 10^{-16}\right)$, a protein involved in ribonuclease reduction $\left(q<5.76 \times 10^{-16}\right)$, and the epsilon subunit of RNA polymerase, recently discovered in
A/T-rich Gram-positive bacteria and thought to protect against phage infection $\left(q<5.71 \times 10^{-15}\right)$. In contrast, COG analysis identified significant enrichment of 5 functional domains associated with spore formation [YlmC $\left(q<1.67 \times 10^{-21}\right)$, SpoIIIAA $\left(q<2.61 \times 10^{-21}\right)$, CwlJ $\left(q<7.18 \times 10^{-20}\right)$, CotJC $(q<$ $\left.7.27 \times 10^{-19}\right)$, and $\left.\operatorname{SpmB}\left(q<5.31 \times 10^{-16}\right)\right]$, as well as irondependent oxidoreductases $\left(q<2.67 \times 10^{-17}\right)$ and aldo/keto oxidoreductases $\left(q<7.85 \times 10^{-21}\right)$ in the gastrointestinal tract (Supplementary Table 7). These results suggest spore formation and oxygen survival, while critical for transmission of gastrointestinal microbiota ${ }^{19}$, are significantly underrepresented in bacteria of the urogenital tract.

Functional enrichment of key metabolic pathways suggests specific nutritional selection on bacteria of the urogenital environment absent in bacteria of the gastrointestinal tract. 


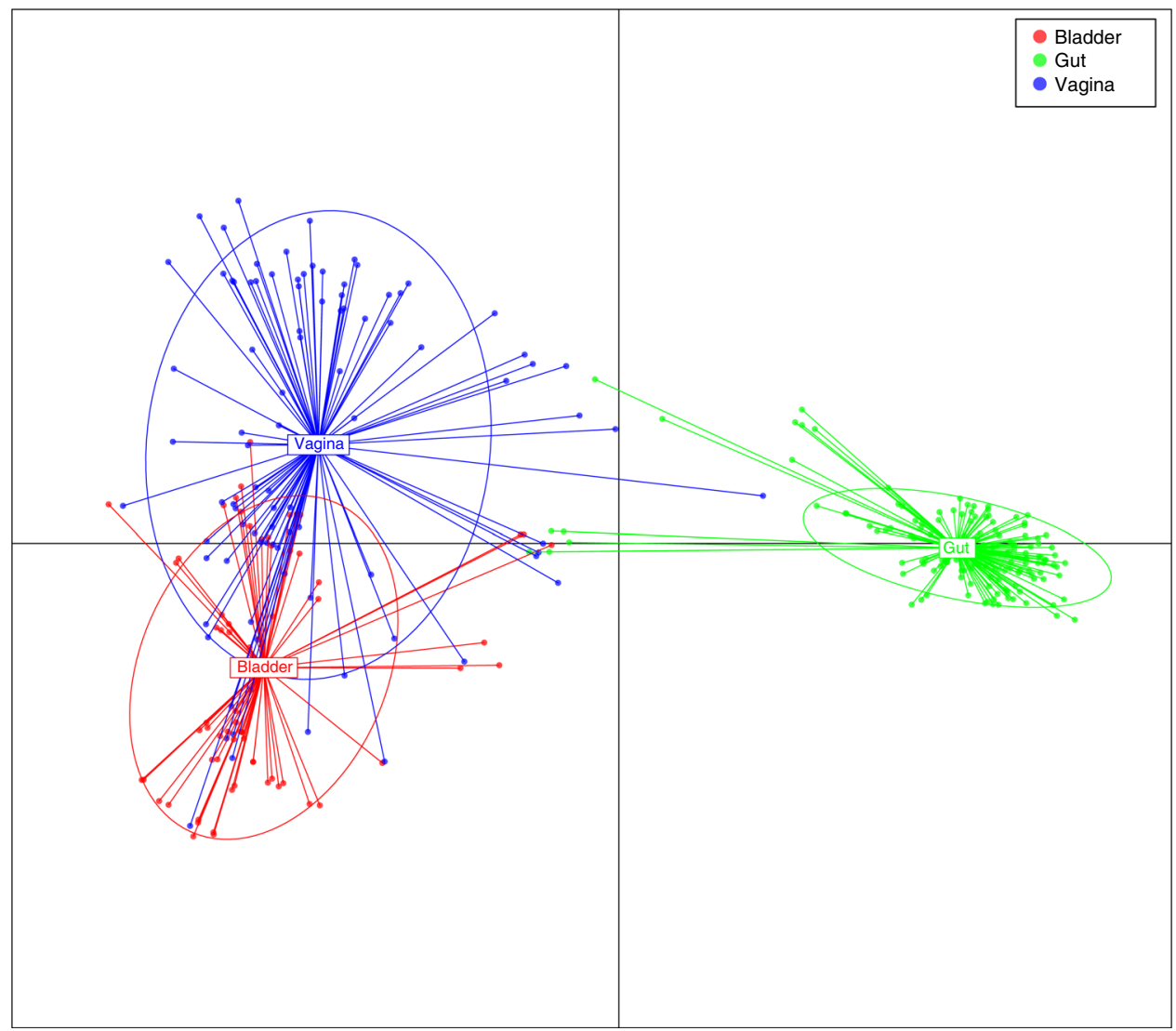

Fig. 2 Functional diversity between genomes of bacterial strains isolated from the bladder, vagina, and intestine. Discriminant analysis of principal components using conserved protein domains (CDD). Each color dot represents a strain from 3 different niches: blue (vagina; $n=92$ ), red (bladder; $n=67$ ), and green (gut; $n=152$ )

Isoprenoids are essential to life, playing indispensable roles in membrane and peptidoglycan biosynthesis and electron transport. The vast majority of bacteria use the methylerythritol phosphate (MEP) pathway for isoprenoid biosynthesis. Intriguingly, this pathway concludes with two Fe-S cluster enzymes ${ }^{20}$. In contrast, the less common mevalonic acid (MVA) pathway, which is enriched in urogenital bacteria Actinomyces neuii, Lactobacillus crispatus, L. gasseri, and L. jensenii, contains no Fe-S clusters. Thus the enrichment of the MVA pathway over the more common MEP pathway may relate to iron availability within the vagina and bladder.

Comparison of vaginal and bladder strains within individual women. Given the significant taxonomic and functional overlap of vaginal and bladder strains in asymptomatic women, we hypothesized the existence of interconnected urogenital microbiota, which we then sought to assess by culturing bacterial strains from the vaginal and bladder microbiota within an independent cohort of women with urgency urinary incontinence symptoms but no clinically detectable urinary infections. Four women contained a species in both anatomical sites (Supplementary Table 8), including $S$. anginsosus, which is associated with urgency urinary incontinence ${ }^{2}$, a putative non-pathogenic $E$. coli, and L. iners and L. crispatus. Both of these Lactobacillus species are associated with health in the vagina ${ }^{21}$, with the latter also associated with the bladder of asymptomatic women ${ }^{2}$.

To determine whether these shared species belong to the same or distantly related bacterial lineages, we next sequenced and compared their genomes to each other and to the genomes of publicly available reference strains (Supplementary Table 9), using the 40-marker gene analysis ${ }^{22,23}$ and ANI analysis. In all the four strain sets tested, the vaginal strain was highly similar to the bladder strain (Fig. 3, blue and red dots). One individual contained E. coli strains that were $99.72 \%$ similar by ANI at both sites. These strains, which occurred in the absence of a clinically diagnosed urinary infection, were most closely related to known commensal strains and lacked both a Type III secretion system and other previously characterized pathogen-associated genes ${ }^{24}$ (Fig. 3a, Supplementary Table 10). The emerging uropathogen $S$. anginosus showed $99.77 \%$ similarity between strains from each site (Fig. 3b, Supplementary Table 11). Finally, the commensal $L$. iners and health-associated L. crispatus strains shared $99.99 \%$ (Fig. 3c, Supplementary Table 12) and 99.80\% (Fig. 3d, Supplementary Table 13) similarity between the vagina and bladder. The existence of these closely related isolates provides strong evidence that bacterial movement between the vaginal and bladder microbiota is not only limited to ascending uropathogenic species, such as $E$. coli, as described previously ${ }^{25}$, but also includes health-associated commensal bacteria.

\section{Discussion}

A growing body of work using catheterized urine in women has found associations between bladder microbiota composition and urgency urinary symptoms, response to anticholinergic medication $^{9}$, risk of postoperative ${ }^{11,26}$ and post-instrumentation UTIs ${ }^{8}$, and kidney stones ${ }^{27}$. Here we provide an extensive, genomesequenced culture collection representing approximately two thirds of bacterial strains detected in the sampled female bladders during both health and disease. Direct cultivation paired with whole-genome sequencing provides the ability to move beyond 


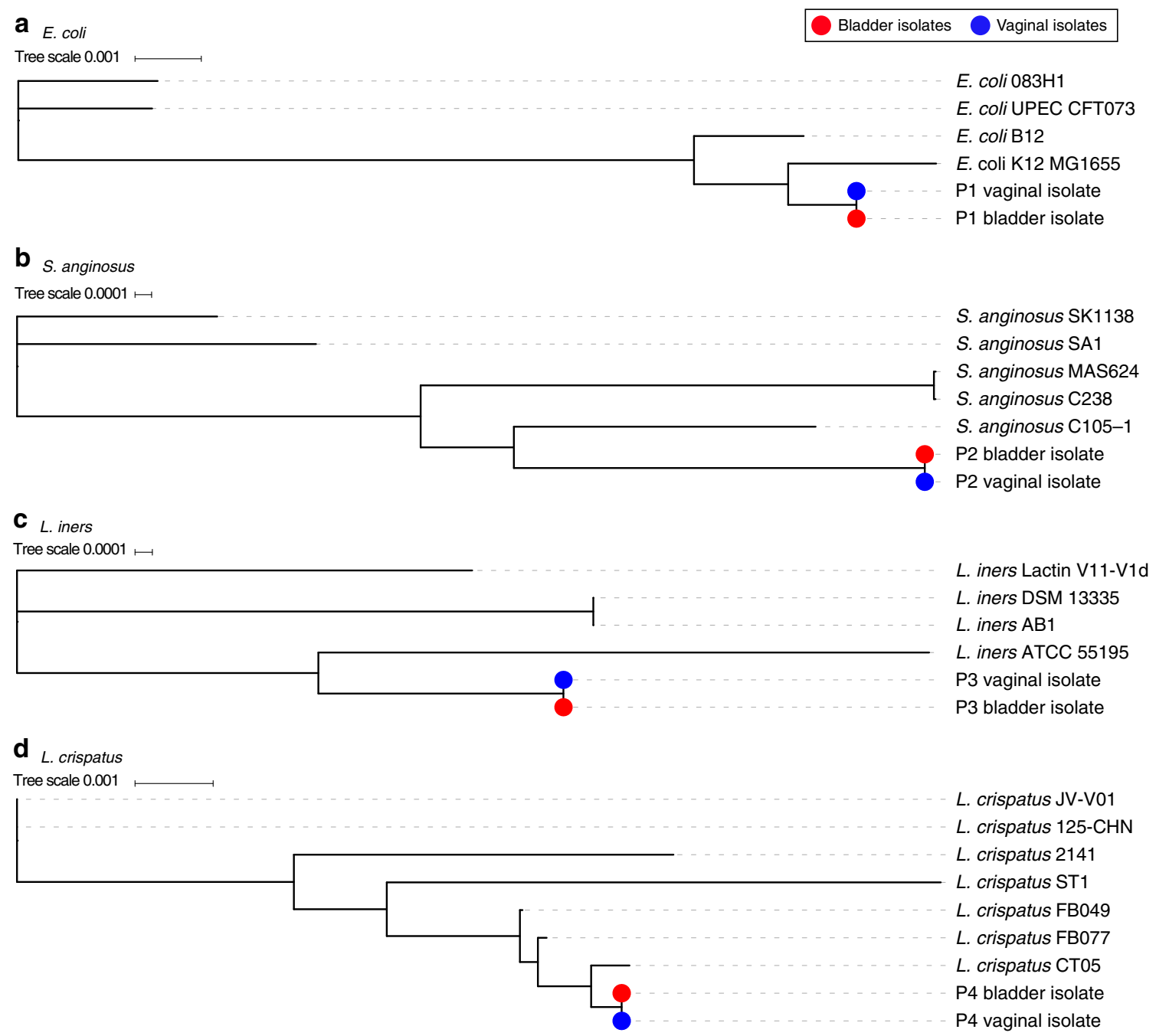

Fig. 3 Phylogenetic comparison of bladder and vaginal strains isolated from individual women using the 40 universal core genes. a Maximum likelihood tree constructed from E. coli strains isolated from the bladder (red) and vagina (blue) of individual patient (P1) and four reference strains. b Maximum likelihood tree constructed from S. anginosus strains isolated from the bladder (red) and vagina (blue) of individual patient (P2) and five reference strains. c Maximum likelihood tree constructed from L. iners strains isolated from the bladder (red) and vagina (blue) of individual patient (P3) and four reference strains. d Maximum likelihood tree constructed from L. crispatus strains isolated from the bladder (red) and vagina (blue) of individual patient (P4) and seven reference strains

broad phylogenetic relatedness to perform strain-level tracking and functional analysis of these microbes and communities. The creation of this comprehensive reference collection, capturing the complete phylogenetic diversity currently cultivated from the bladder, represents a valuable resource to explore the function of both pathogenic and commensal bladder bacteria.

We have demonstrated for the first time that, like some pathogens, highly similar strains of health-associated commensal bacteria are found in both the bladder and vagina of the same individual. Previously, it was thought that a healthy vaginal microbiota was the major factor in preventing ascending infections from migrating into a sterile bladder. The data presented herein suggests that microbial sharing between the vaginal and bladder microbiota is not limited to known and emerging uropathogens, such as E. coli and $S$. anginosus, but also includes health-associated commensal bacteria, such as $L$. iners and $L$. crispatus. Now, we propose that some bacteria that can reside in both the bladder and vagina could provide protection against urinary infection, suggesting that the microbes of these adjacent pelvic floor niches could be considered to be a single urogenital microbiota. This insight, combined with this unique genome- sequenced culture collection, should alter the way we view the bacteria of the female pelvic floor both by enabling further research and by providing new diagnostic and treatment options for UTIs, urgency urinary incontinence, and other associated urinary tract disorders.

\section{Methods}

Patient recruitment. Following Loyola University Medical Center (LUMC) Institutional Review Board approval, participants gave verbal and written consent for chart abstraction and urine collection with analysis for research purposes. Recruitment and urine collection was performed by members of the Loyola Urinary Education and Research Collaborative who are part of the clinical practice of the Female Pelvic Medicine and Reconstructive Surgery Center at LUMC. Exclusion criteria for both cohorts included current UTI (based on urine dipstick) or history of recurrent UTI, antibiotic exposure in the past 4 weeks for any reason, immunologic deficiency, neurological disease known to affect the lower urinary tract, pelvic malignancy or radiation, untreated symptomatic pelvic organ prolapse (POP) greater than POP-Q stage II (vaginal protrusion $>1 \mathrm{~cm}$ outside of the vaginal hymen), or pregnancy.

Patients were recruited as part of separate studies, $2,9,27-29$. In the current study, representative isolates were selected for whole-genome sequencing in order to build as phylogenetically complete a dataset as possible. For this reason, only a few isolates from some patients have been included. For complete understanding of 
each patient's microbiome or for additional metadata, please refer to the primary publications listed in Supplementary Data 1.

Urine collection and EQUC bacterial culturing. Urine was collected aseptically via TUC and was placed in a BD Vacutainer Plus C\&S preservative tube for culturing. Previous work has shown that the microbiota detected in suprapubic aspirates are indistinguishable from the microbiota detected in urine obtain by TUC but distinct from the microbiota in voided urine and vaginal swabs ${ }^{1}$. Thus aspiration and catheterization sample the same niche. Since aspiration bypasses the vulva, vagina, and urethra, this niche must be the bladder. Since it is less invasive than aspiration, TUC is the urine sampling method of choice. We note that because TUC is a transient procedure lasting no more than a few seconds, it is not associated with CAUTI. This study does not use nor address indwelling catheters that, due to biofilm formation, are associated with CAUTIs.

Four patients were chosen to sample both the bladder and vaginal environments (Fig. 3). For these women, the vaginal swab was collected from the posterior fornix prior to the catheterized urine collection.

All samples underwent standard urine culture (SUC) as well as EQUC. For SUC, $1 \mu \mathrm{l}$ of urine was inoculated onto $5 \%$ sheep blood agar plate (BAP) and MacConkey agar plate (BD BBL prepared plated media), incubated aerobically at $35{ }^{\circ} \mathrm{C}$ for $24 \mathrm{~h}$. The detection level was $1000 \mathrm{CFU} / \mathrm{ml}$, represented by 1 colony of growth on either plate. If no growth was observed, the culture was reported as "no growth", indicating no growth of bacteria at the lowest dilution, i.e., 1:1000. SUC results are listed in Supplementary Data 1.

EQUC was performed as described previously ${ }^{3}$. Briefly, $100 \mu$ of urine was grown under five conditions with BD BBL prepared plated media: (1) BAP in $\mathrm{CO}_{2}$ for $48 \mathrm{~h}$, (2) chocolate agar (CHOC) in $\mathrm{CO}_{2}$ for $48 \mathrm{~h}$, (3) colistin and nalidixic acid (CNA) agar in $\mathrm{CO}_{2}$ for $48 \mathrm{~h}$, (4) CDC anaerobe BAP in an anaerobic jar for $48 \mathrm{~h}$, and (5) BAP in aerobic conditions (BD GasPak Anaerobe Sachets) for $48 \mathrm{~h}$. The detection level was $10 \mathrm{CFU} / \mathrm{ml}$, represented by 1 colony of growth on any of the plates. EQUC results are listed in Supplementary Data 1.

Vaginal swabs were collected using BD Liquid Amies Elution Swab (ESwab) collection system. To compare vagina and bladder culture data (Fig. 3, Supplementary Table 9), vaginal samples were cultured using a modified EQUC protocol, with $10 \mu \mathrm{l}$ of urine plated onto BAP and CNA grown for $48 \mathrm{~h}$ in $5 \% \mathrm{CO}_{2}$, and anaerobic BAP grown for $48 \mathrm{~h}$ under anaerobic conditions (BD BBL prepared plated media and BD GasPak Anaerobe Sachets).

Each morphologically distinct colony type was isolated on a different plate of the same medium to prepare a pure culture that was used for identification. Matrixassisted laser desorption ionization-time of flight mass spectrophotometry with the MALDI Biotyper 3.0 software program (Bruker Daltonics, Billerica, MA) was used to identify the bacterial strains, as described elsewhere ${ }^{3}$.

The 149 bacterial strains are available upon request.

Genome sequencing and annotation. The isolates were grown in their preferred medium and pelleted. Genomic DNA was extracted from pelleted cells using a phenol-chloroform method ${ }^{30}$. DNA was prepared and sequenced using the Illumina Hi-Seq platform with library fragment sizes of 200-300 bp and a read length of $100 \mathrm{bp}$ at the Wellcome Sanger Institute, as previously described ${ }^{31}$. Annotated assemblies were produced using the pipeline described previously ${ }^{32}$. For each sample, sequence reads were used to create multiple assemblies using Velvet v1.2 $2^{33}$ and VelvetOptimiser v2.2.5 (https://github.com/tseemann/VelvetOptimiser). An assembly improvement step was applied to the assembly with the best N50 and contigs were scaffolded using SSPACE ${ }^{34}$ and sequence gaps filled using GapFiller ${ }^{35}$. Automated annotation was performed using PROKKA v1.11 ${ }^{36}$.

Whole-genome metagenomic analysis. Whole-genome metagenomic sequencing was performed on the Illumina HiSeq 2500 as described previously ${ }^{18}$ with human contaminating reads filtered by mapping to the Human reference genome (hg19) with bowtie ${ }^{37}$. Filtered sequences were compared at the genus and species levels using lowest common ancestor analysis previously described ${ }^{38}$ and by relative abundance at the sequence level by alignment using the bowtie2 algorithm to the complete bladder culture collection genome catalog.

Phylogenetic analysis and average nucleotide identity analysis. The phylogenetic analysis was conducted by extracting amino acid sequence of 40 universal single copy marker genes ${ }^{22,23}$ from bacterial collection using Spec ${ }^{39}$. The protein sequences were concatenated and aligned with MAFFT v. $7.20^{40}$, and maximumlikelihood trees were constructed using FastTree ${ }^{41}$ with default settings. All phylogenetic trees were visualized in iTOL $^{42}$. ANI was calculated by performing pairwise comparison of genome assemblies using MUMmer ${ }^{43}$.

Functional genomic analysis. To identify protein domains in a genome, we performed RPS-BLAST using $\mathrm{CDD}^{44}$. All protein domains were classified in different functional categories using COG database ${ }^{45}$ and were used to perform $\mathrm{DAPC}^{16}$ implemented in the R package Adegenet v2.0.16. Domain enrichment was calculated using one-sided Fisher's exact test with $p$-value adjusted by Hochberg method in R v3.2.2.
Data availability. Genome and metagenome sequences have been deposited in the European Nucleotide Archive. Accession codes for genome sequences are listed in Supplementary Data 1 and all supplementary tables. Metagenomic sequences are listed in Supplementary Table 1. Other relevant data supporting the findings of the study are available in this article and its Supplementary Information files or from the corresponding authors upon request.

\section{Received: 21 December 2017 Accepted: 26 March 2018}

Published online: 19 April 2018

\section{References}

1. Wolfe, A. J. et al. Evidence of uncultivated bacteria in the adult female bladder. J. Clin. Microbiol. 50, 1376-1383 (2012).

2. Pearce, M. M. et al. The female urinary microbiome: a comparison of women with and without urgency urinary incontinence. MBio 5, e01283-14 (2014).

3. Hilt, E. E. et al. Urine is not sterile: use of enhanced urine culture techniques to detect resident bacterial flora in the adult female bladder. J. Clin. Microbiol. 52, 871-876 (2014).

4. Thomas-White, K., Brady, M., Wolfe, A. J. \& Mueller, E. R. The bladder is not sterile: History and current discoveries on the urinary microbiome. Curr. Bladder Dysfunct. Rep. 11, 18-24 (2016).

5. Whiteside, S. A., Razvi, H., Dave, S., Reid, G. \& Burton, J. P. The microbiome of the urinary tract-a role beyond infection. Nat. Rev. Urol. 12, 81-90 (2015)

6. Kass, E. H. Bacteriuria and the diagnosis of infections of the urinary tract; with observations on the use of methionine as a urinary antiseptic. AMA Arch. Intern. Med. 100, 709-714 (1957).

7. Price, T. K. et al. The clinical urine culture: enhanced techniques improve detection of clinically relevant microorganisms. J. Clin. Microbiol. 54, 12161222 (2016).

8. Pearce, M. M. et al. The female urinary microbiota in urgency urinary incontinence. Am. J. Obstet. Gynecol. 213, 347.e1-347.e11 (2015).

9. Thomas-White, K. J. et al. Incontinence medication response relates to the female urinary microbiota. Int. Urogynecol. J. 27, 723-733 (2015).

10. Thomas-White, K. J. et al. Evaluation of the urinary microbiota of women with uncomplicated stress urinary incontinence. Am. J. Obstet. Gynecol. 216, 55.e1-55.e16 (2016).

11. Thomas-White, K., Fok, C., Mueller, E., Wolfe, A. \& Brubaker, L. Preoperative urinary microbiome reveals post-operative urinary tract infection risk. Neurourol. Urodyn. 34, S21-S22 (2015).

12. Kim, M., Oh, H. S., Park, S. C. \& Chun, J. Towards a taxonomic coherence between average nucleotide identity and 16S rRNA gene sequence similarity for species demarcation of prokaryotes. Int. J. Syst. Evol. Microbiol. 64, 346-351 (2014).

13. Goris, J. et al. DNA-DNA hybridization values and their relationship to whole-genome sequence similarities. Int. J. Syst. Evol. Microbiol. 57, 81-91 (2007).

14. Konstantinidis, K. T. \& Tiedje, J. M. Genomic insights that advance the species definition for prokaryotes. Proc. Natl. Acad. Sci. USA 102, 2567-2572 (2005).

15. Marchler-Bauer, A. et al. CDD: NCBI's conserved domain database. Nucleic Acids Res. 43, D222-D226 (2015).

16. Jombart, T., Devillard, S. \& Balloux, F. Discriminant analysis of principal components: a new method for the analysis of genetically structured populations. BMC Genet. 11, 94 (2010)

17. Faust, K. et al. Microbial co-occurrence relationships in the human microbiome. PLoS Comput. Biol. 8, e1002606 (2012).

18. Browne, H. P. et al. Culturing of 'unculturable' human microbiota reveals novel taxa and extensive sporulation. Nature 533, 543-546 (2016).

19. Browne, H. P., Neville, B. A., Forster, S. C. \& Lawley, T. D. Transmission of the gut microbiota: spreading of health. Nat. Rev. Microbiol. 15, 531-543 (2017).

20. Banerjee, A. \& Sharkey, T. D. Methylerythritol 4-phosphate (MEP) pathway metabolic regulation. Nat. Prod. Rep. 31, 1043-1055 (2014).

21. Gajer, P. et al. Temporal dynamics of the human vaginal microbiota. Sci. Transl. Med. 4, 132 ra52 (2012).

22. Sorek, R. et al. Genome-wide experimental determination of barriers to horizontal gene transfer. Science 318, 1449-1452 (2007).

23. Ciccarelli, F. D. et al. Toward automatic reconstruction of a highly resolved tree of life. Science 311, 1283-1287 (2006).

24. Lloyd, A. L., Rasko, D. A. \& Mobley, H. L. Defining genomic islands and uropathogen-specific genes in uropathogenic Escherichia coli. J. Bacteriol. 189, 3532-3546 (2007)

25. Schwan, W. R. Flagella allow uropathogenic Escherichia coli ascension into murine kidneys. Int. J. Med. Microbiol. 298, 441-447 (2008).

26. Fok, C. S. et al. Day of surgery urine cultures identify urogynecologic patients at increased risk for postoperative urinary tract infection. J. Urol. 189, 1721-1724 (2013) 
27. Barr-Beare, E. et al. The interaction between Enterobacteriaceae and calcium oxalate deposits. PLoS ONE 10, e0139575 (2015).

28. Jacobs, K. M., Thomas-White, K. J., Hilt, E. E., Wolfe, A. J. \& Waters, T. P. Microorganisms identified in the maternal bladder: discovery of the maternal bladder microbiota. AJP Rep. 7, e188-e196 (2017).

29. Dune, T. J. et al. Urinary symptoms and their associations with urinary tract infections in urogynecologic patients. Obstet. Gynecol. 130, 718-725 (2017).

30. Green, M. R., Sambrook, J. \& Sambrook, J. Molecular Cloning: A Laboratory Manual. (Cold Spring Harbor Laboratory Press, Cold Spring Harbor, NY, 2012).

31. Harris, S. R. et al. Evolution of MRSA during hospital transmission and intercontinental spread. Science 327, 469-474 (2010).

32. Page, A. J. et al. Robust high-throughput prokaryote de novo assembly and improvement pipeline for Illumina data. Microb. Genom. 2, e000083 (2016).

33. Zerbino, D. R. \& Birney, E. Velvet: algorithms for de novo short read assembly using de Bruijn graphs. Genome Res. 18, 821-829 (2008).

34. Boetzer, M., Henkel, C. V., Jansen, H. J., Butler, D. \& Pirovano, W. Scaffolding pre-assembled contigs using SSPACE. Bioinformatics 27, 578-579 (2011).

35. Boetzer, M. \& Pirovano, W. Toward almost closed genomes with GapFiller. Genome Biol. 13, R56 (2012).

36. Seemann, T. Prokka: rapid prokaryotic genome annotation. Bioinformatics 30, 2068-2069 (2014).

37. Langmead, B. \& Salzberg, S. L. Fast gapped-read alignment with Bowtie 2. Nat. Methods 9, 357-359 (2012)

38. Forster, S. C. et al. HPMCD: the database of human microbial communities from metagenomic datasets and microbial reference genomes. Nucleic Acids Res. 44, D604-D609 (2016).

39. Mende, D. R., Sunagawa, S., Zeller, G. \& Bork, P. Accurate and universal delineation of prokaryotic species. Nat. Methods 10, 881-884 (2013).

40. Katoh, K. \& Standley, D. M. MAFFT multiple sequence alignment software version 7: improvements in performance and usability. Mol. Biol. Evol. 30, 772-780 (2013).

41. Price, M. N., Dehal, P. S. \& Arkin, A. P. FastTree: computing large minimum evolution trees with profiles instead of a distance matrix. Mol. Biol. Evol. 26, 1641-1650 (2009).

42. Letunic, I. \& Bork, P. Interactive Tree Of Lifev2: online annotation and display of phylogenetic trees made easy. Nucleic Acids Res. 39, W475-W478 (2011).

43. Delcher, A. L., Phillippy, A., Carlton, J. \& Salzberg, S. L. Fast algorithms for large-scale genome alignment and comparison. Nucleic Acids Res. 30, 2478-2483 (2002).

44. Marchler-Bauer, A. et al. CDD: a conserved domain database for interactive domain family analysis. Nucleic Acids Res. 35, D237-D240 (2007).

45. Tatusov, R. L., Galperin, M. Y., Natale, D. A. \& Koonin, E. V. The COG database: a tool for genome-scale analysis of protein functions and evolution. Nucleic Acids Res. 28, 33-36 (2000).

46. Jombart, $\mathrm{T}$. adegenet: a $\mathrm{R}$ package for the multivariate analysis of genetic markers. Bioinformatics 24, 1403-1405 (2008).

\section{Acknowledgements}

This work was supported by the Wellcome Trust (098051), the United Kingdom Medical Research Council (PF451 to T.D.L.), the Australian National Health and Medical Research Council (1091097 to S.C.F.), the Victorian Government's Operational Infrastructure Support Program (S.C.F.), and an NIH grant (R01 DK104718 to A.J.W.). We would also like to acknowledge sequencing and bioinformatics support from the Wellcome Sanger Pathogen Informatics and sequencing team. Finally, for prior patient recruitment, we want to acknowledge the Loyola Urinary Education and Research Collaborative (LUEREC), specifically Mary Tulke RN and Dr. Linda Brubaker, Dr. Elizabeth Mueller, Dr. Cynthia Brincat, Dr. Susanne Taege, and Dr. Tanaka Dune, and the patients who provided the samples for this study.

\section{Author contributions}

K.T.-W., S.C.F., N.K., A.J.W., and T.D.L. designed the study and analyzed the data; K.T.W., M.V.K., E.E.H., and T.K.P. carried out culturing, bacterial isolation, and DNA extraction; C.P. aided in genome analysis; M.D.S. managed samples and genome sequencing. All authors contributed to manuscript preparation.

\section{Additional information}

Supplementary Information accompanies this paper at https://doi.org/10.1038/s41467018-03968-5.

Competing interests: The authors declare no competing interests.

Reprints and permission information is available online at http://npg.nature.com/ reprintsandpermissions/

Publisher's note: Springer Nature remains neutral with regard to jurisdictional claims in published maps and institutional affiliations.

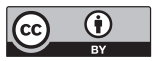

Open Access This article is licensed under a Creative Commons Attribution 4.0 International License, which permits use, sharing, adaptation, distribution and reproduction in any medium or format, as long as you give appropriate credit to the original author(s) and the source, provide a link to the Creative Commons license, and indicate if changes were made. The images or other third party material in this article are included in the article's Creative Commons license, unless indicated otherwise in a credit line to the material. If material is not included in the article's Creative Commons license and your intended use is not permitted by statutory regulation or exceeds the permitted use, you will need to obtain permission directly from the copyright holder. To view a copy of this license, visit http://creativecommons.org/ licenses/by/4.0/.

(C) The Author(s) 2018 